\title{
MEDIEN, UNIVERSITÄTEN, LÜGEN Ein Kommentar zum Beitrag von Bernhard Pörksen
} von CLAUS PIAS

I.

Die wohl unterhaltsamste Passage von Neil Postmans Bestseller Wir amüsieren uns zu Tode befindet sich genau in der Mitte des Buches und bildet dessen dramatischen Höhepunkt. Um die These vom Entertainment als «Superideologie des gesamten Fernsehdiskurses» ${ }^{1}$ zu dramatisieren, wählt er ein Beispiel von größtmöglicher Fallhöhe, das allen damaligen Leser_innen bekannt gewesen sein dürfte. Es ist die Fernsehdiskussion nach The Day After (Regie: Nicholas Meyer, USA 1983), einer der publikumsreichsten Erstausstrahlungen der gesamten Fernsehgeschichte. Hochkarätig besetzt mit Talkgästen wie Carl Sagan, Henry Kissinger, Elie Wiesel, Robert McNamara, George Shultz und anderen bildete sie das Finale eines über Monate währenden Medienereignisses, bei dem es (an einem Höhepunkt der Friedensbewegung und nach dem Scheitern der Genfer Verhandlungen) um nichts Geringeres ging, als um den bald zu erwartenden Atomkrieg und das Ende der Menschheit. Postmans Kunstgriff besteht darin zu zeigen, dass nicht einmal der Ernst dieser 〈Lage> ausreicht, um eine Diskussion zu ermöglichen, die diesen Namen verdient und die zumindest ansatzweise zur Buchkultur und der amerikanischen Rhetoriktradition aufschließen kann. Über etliche Seiten entfaltet sich seine Beschreibung von Details wie Carl Sagans Frisur und (ausbleibendem) Rollkragenpullover bis hin zur Gesamtsituation, dass «sprachgewandte Männer mit großer politischer Urteilskraft» ${ }^{2}$ vorgeführt werden, als seien sie bei einem Schönheitswettbewerb in Las Vegas. Und dies alles nur, um zu zeigen, dass Denken im Fernsehen gar nicht stattfinden kann - und zwar ungeachtet, wie wichtig das Thema, wie lauter die Absichten der Beteiligten und wie groß ihre Kompetenz auch sei; sondern aus rein medienspezifischen Gründen.

Dies wäre nicht erwähnenswert, würde der Beitrag von Bernhard Pörksen in diesem Heft (der auf Postman verweist) nicht genau diesem Strukturmodell $<$ klassischer $>$ Medientheorie folgen. Dieses funktioniert erstens durch die Feststellung von Dringlichkeit: Auf dem Spiel stehen akut «die Verständigungsfähigkeit

1 Neil Postman: Wir amüsieren uns zu Tode, Frankfurt/M. 2008, 110. 2 Ebd., 114. 
und die Wertebindung der Gesellschaft» und damit das Politische selbst. Zweitens durch das Erschrecken eines Evidenzverlusts: Es ist eine mediale «Simulationsmaschinerie», die «nackte Machtinteressen» camoufliert. Drittens durch die Zuschreibung von Verantwortlichkeit an Medien: Tweets und sogenannte soziale Medien bilden die Möglichkeitsbedingung einer «Mischung aus Relativismus und brutaler Machtpolitik». Und viertens durch ein medienhistorisches Verlustnarrativ, das neue an alten Medien bemisst: Die «Destabilisierung der Printmedien» und das Ende der «Qualitätspublizistik» machen einer «Gleichwertigkeitsdoktrin» digitaler Medien Platz.

Dieses diskursstrategische Geviert lässt sich in verschiedenen Szenen der Medientheorie-Geschichte des 20. Jahrhunderts rekonstruieren - etwa in Karl Kraus' Pressekritik des <Impressionistisch-Werdens> der Nachricht, in Günther Anders' Fernsehkritik des Erfahrungsverlusts und der Zurichtung von Wirklichkeit oder in Vilém Flussers Bildkritik des Verlusts von Argument und Geschichtlichkeit. Erstens sind es stets medientechnologische Umbrüche, die den Grund von und den Appell zu Medientheorie bilden, die sie hervorrufen und provozieren. Durch dieses Dringlichkeitsargument schaffen sich Medientheorien selbst den Ort ihrer Geltung. Zweitens geht deren Erscheinen mit einer Fremdheitserfahrung und einer Unterbrechung kultureller Routinen einher, deren Artikulation Medientheorien oft in die Rolle des Querulanten innerhalb der akademischen Arbeitsteilung und der Disziplinensystematik verwiesen hat. Von dieser Position aus betreiben sie umfassende Gesellschaftskritik, die als Medienkritik reformuliert wird. Diese wiederum hängt drittens von einer vorgängigen Kodifizierung sogenannter Leitmedien ab, denen Verantwortung flexibel zugeschrieben werden kann. In diesem Sinne war Medientheorie immer gezwungen, heterogenes Belegmaterial zu rekrutieren, und gestaltete sich notwendig <dilettantisch〉. Viertens impliziert diese verstreute Kausalitätsbehauptung eine Epochalisierung: Kulturtechniken, Mentalitäten (und damit auch Medientheorien selbst) stellen sich selbst als Produkte bestimmter Medienepochen dar. Klassische Medientheorien favorisieren daher die Autor_ innenposition des <letzten Zuschauers〉 - die eines_einer Beobachter_in im Dazwischen, der_die im Moment des Umbruchs noch zu erkennen vermag, was gerade verschwindet, und noch vom Untergang berichten kann.

Während man (schon aus wissenschaftspolitischen Gründen) kaum bereit sein wird, das Dringlichkeitsargument zu revidieren, wären die weiträumigen (und tatsächlich offenen) Fragen, wie man mit dieser medientheoretischen und medienkritischen Tradition umgehen möchte, die ja lange Zeit auch unabhängig von der Existenz einer disziplinären Medienwissenschaft praktiziert wurde und wird. Solche Fragen beträfen etwa die Notwendigkeit der Annahme von <Leitmedien> und der ihnen unterstellten Homogenität, die je nach Wahl des Beobachtungsausschnitts einzelner Phänomene zerfallen dürfte. Gibt es überhaupt <die> digitalen Medien? Sie beträfen darüber hinaus das Thema jener Kausalitätszumutung, die die Bedeutung der Medien der Medientheorie je 
erst konstituiert. Wie viel ist man eigentlich bereit, <den Medien> zuzumuten? Und sie beträfen zuletzt die Frage der Epochalisierung und damit die <Trauer der Vollendung $>$ bestimmter Medienkulturen, auf die Hans Blumenberg einmal lapidar antwortete, es gebe keine Augenzeugen von Epochenumbrüchen. ${ }^{3}$ Lassen sich vielleicht diagnostische <Gegenbegriffe $>$ finden, die nicht eines Verlustnarrativs bedürfen?

\section{II.}

Bernhard Pörksens Beitrag ist offensichtlich ein Appell: Er endet mit einem Aufruf zur Wirksamkeit außerhalb der Universität, mit der Forderung ein «Schulen- und Schurkendenken» zu verabschieden, für «den Wert einer offenen Gesellschaft» einzutreten und eine «gemeinsame Vision, eine echte Aufgabe [für ...] die Geistes- und Sozialwissenschaften» zu entwickeln, die diese wieder an die «Dramen der wirklichen Welt heranführen» möge. Damit lokalisiert sich der Text nicht allein in der Gattung der Medien-, sondern auch der Universitätskritik. ${ }^{4}$ Seine Argumentation entfaltet sich ausgehend von der Diagnose einer doppelten Entwendung des konstruktivistischen Projekts, die von ihrer Anlage her bekannt ist. ${ }^{5}$ Sie handelt einerseits von der Trauer, dass sich Abweichung in Normativität verwandelt habe, Antiautoritäres zur neuen Autorität aufgestiegen sei, Anti-Orthodoxie in Orthodoxie und Skepsis in Dogmatismus umgeschlagen sei. ${ }^{6}$ Oder mit den Worten von Geoffrey Winthrop-Young: Paris ist auch nicht mehr das, was es mal war. ${ }^{7}$ Andererseits handelt sie von der Wut, dass und wie konstruktivistische Theoreme (hier als «postmodern» zusammengefasst) von einer populistischen «Gegenseite〉 nach Belieben gekapert werden können ${ }^{8}$ - sei es in Form der Verschleierung finanzieller, politischer oder militärischer Interessen oder sei es in Form der Zuschreibung einer Macht, die der Konstruktivismus selbst nie haben wollte, nämlich (vermeintlich) als philosophischer Berater für Phänomene wie Fake News zu gelten.

So sehr man diesem Argument auch zugeneigt sein mag, so sehr drängen sich doch drei Beobachtungen auf: Erstens hinterlässt der Beitrag (trotz seiner kämpferischen Entschlossenheit) eine leichte Ungewissheit, wie man mit dieser Situation nun umgehen soll - eine Ungewissheit, die nicht zuletzt Züge der beschriebenen Konfusion der <Fronten> selbst trägt. Denn wie würde die geforderte «Erkenntnistheorie des Widerstands» aussehen? Und wo würde sie stattfinden? Offensichtlich will sie sich nicht dem Neuen Realismus ergeben und dennoch einer «Sehnsucht nach Verbindlichkeit» nachkommen. Sie will dem ursprünglichen Gestus des Konstruktivismus treu bleiben und dennoch an die «Dramen der wirklichen Welt heranführen», die Rhetorizität von Wahrheitsansprüchen bloßlegen und zugleich in bestimmten Kontexten auf so etwas wie <Tatsachenwahrheiten $>$ insistieren. Geht es also um so etwas wie «strategic essentialism» (Gayatri Chakravorty Spivak) in Fragen der Beobachtung? Um eine Kasuistik des Konstruktivismus, wie der Überzeugungsversuch Heinz von Foersters
3 Vgl. Hans Blumenberg: Legitimität der Neuzeit, Frankfurt/M. 1996, 545 .

4 Erhellend dazu Stefan Collini: Speaking of Universities, London/New York 2017.

5 Wie eminent die Frage nach ‘Ermüdungserscheinungen` des Konstruktivismus ist, bezeugt die Existenz eines DFG-Graduiertenkollegs ( DDas Reale in der Kultur der Moderne"), das sich bereits seit 2010 um Alternativen bemüht.

6 Für ein vergleichbares Phänomen vgl. als Vorschlag einer Historisierung und Systematisierung David Kaldewey: Der Campus als 'Safe Spaces. Zum theoretischen Unterbau einer neuen Bewegung, in: Mittelweg, Nr. 36, H. 26/4-5, 2017, 132-153.

7 Vgl. Geoffrey Winthrop-Young: What was Kittler's Media Theory?, Vortrag auf dem Colloquium The Sirens Go Silent, New York, 16.3.2013, youtu.be/DDUNszH_FWE, gesehen am 29.6.2018.

8 Vgl. prominent dazu Bruno Latour: Das Elend der Kritik, Zürich, Berlin 2007. 
9 Vgl. Hanna Engelmeier, Philipp Felsch: «Gegen die Uni studieren?. Ein Vorwort, in: Mittelweg Nr. 36 , H. 26/4-5, 2017, 4-13.

10 Collini: Speaking of Universities, 62.

11 Engelmeier, Felsch: «Gegen die Unir, 5 f.

12 Etwa im Sinne von Jean François Lyotard: Die Moderne redigieren, Bern 1988. nahelegen könnte? Und umgekehrt könnte man fragen: Woher kommt die Gewissheit, dass diese Erkenntnistheorie so erfolgreich wäre, wenn sie nur erst den Seminarraum verlassen hätte? Zumal wenn man umgekehrt konstatiert, dass akademische Milieus ohnehin machtlos sind und ihr Einfluss (wie polemisch gezeigt) maßlos überschätzt wird? Zumal man aus systemtheoretischer Perspektive ohnehin damit umzugehen hätte, dass Wissenschaft und Politik als Systeme mit unterschiedlichen Leitdifferenzen aneinander vorbei umwelten. Der Text scheint sich damit streckenweise in den Widerspruch zu verstricken, den er analysiert: Auf der einen Seite <Theorie〉, die nur das Gespinst einer Minderheit im Elfenbeinturm sei, auf der anderen Seite aber $\langle$ Theorie>, die durch ihre breite gesellschaftliche Durchsetzung enormen politischen Flurschaden anrichtet. Beides ist, so könnte man behaupten, von ebenso stillschweigenden wie tradierten Vorannahmen über Rolle und Funktion der Universität getragen, insofern man ihr entweder nichts zutraut oder ihr zu viel zumutet.

Zweitens könnte man daher (auf die Gattungsfrage zurückkommend) behaupten, dass Pörksens Diskussionsbeitrag eine antiakademische Sprecher_ innenposition beansprucht, wenn man Antiakademismus als das eingeschlossene Ausgeschlossene der Universität versteht. Denn wie kaum eine andere Institution kapitalisieren Universitäten (und zwar ideologisch wie finanziell) genau diejenige Kritik, die auf ihre eigene Abschaffung zielt. ${ }^{9}$ Dieser Mechanismus ist ihre Stärke und zugleich Blindheit, und er verläuft (wie Stefan Collini bemerkt hat) in Rhythmen von Reformtrauer: «[T]he alien measures which each generation of champions of the <idea of the university> complain about are usually introduced by statements from politicians or administrators that at least pay lipservice to the diluted version of the day before yesterday's <idea of the university> literature.» ${ }^{10}$ Insofern könnte man die Widersprüche des Textes auf eine bestimmte Sprecher_innenposition zurückführen. Diese wäre dem ersten Typus von Hanna Engelmeiers Taxonomie des historischen Antiakademismus zuzuschlagen: eine in progressiven wie konservativen Formen anzutreffende Kritik, die «mit der Sorge gepaart [ist], dass die Universität ihren Auftrag, der Wahrheitsfindung und Nützlichkeit einer wie immer zu beschreibenden Gesellschaft zu dienen, nicht mehr erfüllen kann. Sie verbindet sich [...] mit nostalgischen Wünschen, in eine möglicherweise nur in der Fantasie vorhandene Universität [...] zurückzukehren.» ${ }^{11}$

Drittens könnte man die rezenten Schuld- oder Wirkungszuweisungen einer sogenannten Postmoderne auch als Chance zu deren historischer Aufarbeitung begreifen. ${ }^{12}$ Denn was an den Diskussionen, die ja weit über den vorliegenden Beitrag hinaus geführt werden, auffällt, ist deren Verengung der postmodernen Agenda auf einige erfolgreiche geistes- und kulturwissenschaftliche Bewegungen oder Kritiken wie Dekonstruktion, Konstruktivismus oder Medientheorie. Diese Reduktion ist ebenso schmeichelhaft wie falsch. Sie spricht den Geistesund Kulturwissenschaften eine Bedeutung zu, die sie generell sicher gerne besäßen, die sie aber in ihren individuell unterstellten Folgen (z. B. Donald Trump 
als «postmoderne[r] Herrscher») genauso entschlossen zurückweisen müssen. Was die Reduktion falsch macht, ist die rückblickende Unterschlagung des gesamten Diskurses um ein postindustrielles Zeitalter, der sich eben nicht in den Kultur- und Geisteswissenschaften, sondern seit den r96oer Jahren in Wirtschaft, Politikberatung, Soziologie und Technowissenschaften herausgebildet hat. ${ }^{13}$ Sucht man eine gesellschaftspolitisch einflussreiche postmoderne Agenda, wäre sie wohl eher dort zu finden - in einer antikommunistischen Strategie, die dazu diente, den <Wettlauf der Systeme> zu gewinnen, indem man sich auf ein anderes Spielfeld begibt. Die Verantwortung für die Gegenwart an eine rein geistes- und kulturwissenschaftliche Postmoderne zu delegieren, ignoriert insofern nur die Notwendigkeit, diese als Seitenzweig eines viel umfassenderen Diskussionszusammenhangs zu realisieren. Und man könnte durchaus fragen, wem diese Verengung nutzt.

\section{III.}

Neben den Motiven von Medien- und Universitätskritik ist interessant, welche Vorannahmen in der Beobachtung des Phänomens Fake News enthalten sind. Jürgen Kaube etwa hat Verwunderung über die Empörung geäußert, weil sie die implizite Annahme enthält, dass im politischen System zuvor immer <Wahrheit> geherrscht habe. ${ }^{14}$ Dies ähnelt der klassischen Diagnose Hannah Arendts: «[G]ezielte Irreführungen und blanke Lügen als legitime Mittel zur Erreichung politischer Zwecke kennen wir seit den Anfängen der überlieferten Geschichte. Wahrhaftigkeit zählte niemals zu den politischen Tugenden, und die Lüge galt immer als ein erlaubtes Mittel in der Politik. ${ }^{15}$ Möglicherweise ist es eine Frage von Art und Ausmaß, gepaart mit einem Interesse am Neuen digitaler Medien, die diese historische Evidenz zeitweilig vergessen gemacht hat. Sicherlich aber taugt sie dazu, gewisse Grundannahmen zu historisieren, die den stillschweigenden Grund zu Empörung und Appell bilden. Denn die Wahrheits- und Wahrhaftigkeitspflichtigkeit des Politischen ist als historisch kontingenter Kern der Argumentation relativ jung. Sie verweist auf die modernen, demokratischen Prinzipien von Transparenz und Kontrolle, die sich im Zuge der Aufklärung etabliert haben. So hatte Immanuel Kant in seiner kleinen Schrift Über ein vermeintes Recht aus Menschenliebe zu lügen selbstbewusst für die Praxistauglichkeit des kategorischen Imperativs und für eine Politik des weltbürgerlichen Vernunftrechts plädiert. ${ }^{16}$ Dieses Projekt eines rigorosen politischen Lügenverbots ist seitdem vielfach diskutiert worden. ${ }^{17}$ In seiner antimachiavellistischen Stoßrichtung ist es Produkt einer Kultur der Aufrichtigkeit, die die bis dahin vorherrschenden politischen Klugheitsregeln von simulatio und dissimulatio desavouiert und mit moralischen Begriffen wie Heuchelei, Lüge oder Verleumdung belegt hat. ${ }^{18}$ Bis in die Wortwahl hinein wiederholen die heutigen Vorwürfe diese Umwertung - etwa wenn von der «Simulationsmaschinerie» Putins die Rede ist, die mit «nackte[n] Machtinteressen» einhergehe.
13 Vgl. etwa Zbigniew Brzezinski: Between Two Ages: America's Role in the Technetronic Era, New York 1970; Daniel Bell: The Coming of the Post-Industrial Society, New York 1973; Alain Touraine: The Post-Industrial Society. Tomorrow's Social History: Classes, Conflicts and Culture in the Programmed Society, London 1971. Die Kenntnis dieses Diskussionszusammenhangs wird noch explizit in Jean François Lyotard: La Condition postmoderne: Rapport sur le savoir, Paris 1979.

14 Vgl. Jürgen Kaube: Was tun gegen (Fake-News'? Die Realität ist nicht konsenspflichtig, in: Frankfurter Allgemeine Zeitung, dort datiert 17.1.2017, faz.net|-gqz-8qftl, gesehen am 12.7.2018.

15 Hannah Arendt: Die Lüge in der Politik, in: dies.: Wahrheit und Lüge in der Politik. Zwei Essays, München 1972, 7-43, hier 8.

16 Vgl. Immanuel Kant: Über ein vermeintes Recht, aus Menschenliebe zu lügen, in: Kleinere Schriften zur Geschichtsphilosophie, Ethik und Politik, hg. v. Karl Vorländer, Hamburg 1973, 199-206.

$17 \mathrm{Vgl}$. Georg Geismann, Hariolf Oberer (Hg.): Kant und das Recht der Lüge, Würzburg 1986.

$18 \mathrm{Vgl}$. Wolfgang G. Müller: Ironie, Lüge, Simulation, Dissimulation und verwandte rhetorische Termini, in: Christian Wagenknecht (Hg.): Zur Terminologie der Literaturwissenschaft, Stuttgart 1988, 189-208. 
19 Dies wurde innerhalb der hier verhandelten ‘Postmoderne» durchaus unternommen, etwa in prominenten Rückgriffen auf das Mittelalter oder auf die Wunderkammer.

20 Eine solchen Versuch der experimentellen Verschränkung organisationssoziologischer Gegenwartsbeobachtungen mit vormodernen Begriffen und Konzepten haben wir zur Diskussion gestellt in Timon Beyes, Claus Pias: Transparenz und Geheimnis, in: Zeitschrift für Kulturwissenschaften, Nr. 8, H. 2, 2014, 111-130.

21 Zur Dissimulation als Praxis in Diplomatie, Politik und Intimität vgl. Jean-Pierre Cavaillé: Dis/simulations. Jules-César Vanini, François la Mothe le Vayer, Gabriel Naude, Louis Machon et Torquato Accetto, Paris 2002.

22 Vgl. Herfried Münkler: Machiavelli. Die Begründung des politischen Denkens der Neuzeit aus der Krise der Republik Florenz, Frankfurt/M. 1984.

23 Vgl. Herfried Münkler: Moralvirtuosen sind gefährlich, in: Brand Eins, Nr. 9, 2011, 88-93.

24 Torquato Acetto: Della dissimulazione onesta [1641], in: Benedetto Croce, Santino Caramella (Hg.): Politici e moralisti del seicento, Bari 1930, 143-173. Dank an Horst Bredekamp für diesen Hinweis.
Eine Reflexionsmöglichkeit bestünde also darin, nicht den modernen Reflexen zu folgen, sondern gerade umgekehrt die vormodernen Begriffe ernst zu nehmen. Wenn es tatsächlich so ist, dass die Gegenwart von einem epochalen Wandel der Medienkultur gekennzeichnet ist, an dessen Beschreibung moderne Begriffe und Konzepte stumpf werden, dann braucht es vielleicht andere <Gegenbegriffe〉. Und wenn die modernen Begriffe zunehmend anachronistisch werden, gälte es vielleicht, diesen Anachronismus gerade zu forcieren und auf vormoderne Begriffe zurückzugreifen. ${ }^{19}$ Es ginge dabei nicht darum, deren Geltung zu behaupten, sondern darum, sie für ein Gedankenexperiment fruchtbar zu machen, das die Frage der Epochalität unserer gegenwärtigen (digitalen) Kultur hervortreibt. ${ }^{20}$

In diesem Sinne bieten die Klugheitslehren der Renaissance und des Barock einen reichen Vorrat an Philosophie, Theorie und Kasuistik der politischen Lüge, der auf die berühmte Formel qui nescit dissimulare nescit regnare zu bringen ist. ${ }^{21}$ Der Rechtfertigungsansatz für die politische dissimulatio (wie er prominent etwa bei Machiavelli artikuliert ist) liegt dabei in der Staatsräson, d.h. im Zusammenhalt und der Bewahrung gesellschaftlicher Ordnung. Diese zwingt den Fürsten, sich so zu verhalten, wie sich der Einzelne nicht verhalten sollte. Die Ethik des Amtes dominiert die Ethik des Individuums, insofern sie Privatperson und Amtsinhaber_in trennt. ${ }^{22}$ Oder anders herum: Die Kunst des Betrugs steht im Zeichen einer höheren Wahrheit; sie ist nicht Selbstzweck, sondern an immer schon moralisch ausgestattete Ziele geknüpft (auch wenn wir diese heute nicht teilen würden). In diesem Sinne ist Machiavelli als Realist und Physiker republikanischer Politik interpretiert worden, der eine pragmatische Kategorisierung von Methoden vornimmt und das Handeln politischer Akteur_innen von Intentionalität auf Funktionalität umstellt. ${ }^{23}$ Zugleich hat die dissimulatio als literarische Strategie des Fingierens eine eminent ästhetische Dimension. Nicht nur im Kontext der Politik, sondern auch in dem der Moralistik der höfischen Gesellschaft gilt daher eine Logik der «ehrenwerten Verhehlung» und des «tugendhaften Betrugs», die ihre Praktikant_innen nicht zuletzt vor der unerträglichen Nacktheit der Tugend ${ }^{24}$ schützt. An solche Annahmen schloss sich eine umfangreiche Kasuistik guter und schädlicher simulatio und dissimulatio an, deren genauere Betrachtung für die Gegenwartsbeschreibung fruchtbar gemacht werden könnte - nicht zuletzt wenn es wie in Pörksens Beitrag um fallweise Abwägungen der Gültigkeit und Reichweite konstruktivistischer Epistemologien geht.

Für einen solchen Blickwechsel auf die Lüge als Normalfall (und nicht als Ausnahmefall) des Politischen, könnten mindestens zwei Referenzen hilfreich sein, in deren Denken die vormoderne Komplexität und Funktionalität der Lüge präsent ist.

Einerseits die bereits zitierte Hannah Arendt, die im Anschluss an Leibniz verschiedene, je eingeschränkte Wahrheitstypen unterscheidet: «Wenn politische Macht sich an Vernunftwahrheiten vergreift, so übertritt sie gleichsam 
das ihr zugehörige Gebiet, während jeder Angriff auf Tatsachenwahrheiten innerhalb des politischen Bereichs selbst stattfindet.» ${ }^{25}$ Gegen Kants Rigorismus bringt Arendt die Lüge hier als notwendigen Bestandteil des Politischen (wenn nicht gar dessen Wesen) in Anschlag, was umgekehrt bedeutetet, dass (absolute) Wahrheitsansprüche «vom Standpunkt der Politik [aus] gesehen [...] despotisch» sind. ${ }^{26}$ Dies schließt Kritikfähigkeit - im Falle Arendts etwa der Vertuschungs- und Täuschungsversuche der US-Regierung während des Vietnamkrieges - eben nicht aus, sondern ein.

Andererseits aber wäre Niklas Luhmann anzuführen, der mit seiner Systemtheorie an vormoderne Wahrheitsdiskurse des Politischen anschließen kann, weil sich die Codierung des Funktionssystems Politik nicht mit dem Moralcode gut/schlecht gleichsetzen lässt. «Mein Eindruck [so Luhmann] ist, dass nur damals das Problem der Ehrlichkeit in der Politik wirklich ernst genommen und diskutiert worden ist». ${ }^{27}$ Autoren wie Accetto, Bacon oder Machiavelli sind systemtheoretisch deshalb reizvoll, weil sie zwei Paradoxien artikulieren: die des Moralcodes und die der Kommunikation.

Bei der Paradoxie des Moralcodes geht es darum, dass die Moral gelegentlich unmoralisches Handeln erfordert, wenn sie sich nicht selbst unmöglich machen will. Bei der Paradoxie der Kommunikation geht es um die Kommunikation von Nichtkommunizierbarem. Die Vergeblichkeit des Sichabmühens an diesen beiden Paradoxien hat zu hochartifiziellen Konstrukten geführt, die zu dem Besten gehören, was man über unser Thema finden kann. ${ }^{28}$

Da diese jedoch seit Kant nicht mehr in den Kategorien der prudentia verhandelt werden können, bleibe - so Luhmann - nur noch Naivität oder Zynismus im Umgang mit Moral übrig. Wo das Projekt der Aufklärung gescheitert sei, weil es eine «moralische Integrierbarkeit der Gesellschaft» voraussetzte und heute «niemand, der sich für Moral einsetzt, in Anspruch nehmen kann, die Gesellschaft zu vertreten», werden die vormodernen Kasuistiken wieder relevant: «Eine [politische] Ethik, die modernen gesellschaftlichen Verhältnissen gerecht werden will, müßte über die Anwendung und Nichtanwendung von Moral befinden können. [Und sie ...] hätte vor allem diese Eigenregie des Systems zu reflektieren ${ }^{29}$
25 Hannah Arendt: Wahrheit und Politik, in: dies.: Wahrheit und Lüge, 44-92, hier 49 .

26 Ebd., 61.

27 Niklas Luhmann: Die Ehrlichkeit der Politiker und die höhere Amoralität der Politik, in: Detlef Horster (Hg.): Die Moral der Gesellschaft, Frankfurt/M. 2008, 163-174, hier 164 .

28 Ebd., 166.

29 Ebd., 174, 168. 